\title{
3,5-二取代的异噁唑啉类衍生物的合成及杀菌活性研究
}

\author{
宁国慧 ${ }^{a}$ 赵温涛 ${ }^{a}$ 边 强 ${ }^{b}$ 唐向阳*, \\ $\left({ }^{a}\right.$ 天津大学理学院化学系 天津 300072) \\ ( ${ }^{b}$ 南开大学国家农药工程研究中心 天津 300071)
}

\begin{abstract}
摘要 取代苯甲醛与盐酸羟胺反应生成取代苯甲醛肜, 经氯代得到相应的氯代苯甲醛肜, 再和 $N$-乙烯基吡咯焢酮、2乙烯基吡啶、4-乙烯基吡啶等进行 1,3-偶极环加成反应，合成了 15 个未见报道的异㯺唑啉类化合物. 其结构经过 ${ }^{1} \mathrm{H}$ $\mathrm{NMR},{ }^{13} \mathrm{C}$ NMR 及高分辨质谱表征. 初步的生物活性测试结果表明, 目标化合物 $\mathbf{3 b}$ 对黄瓜灰霉表现出了很好的防效, 化合物 $3 \mathrm{c}$ 对番茄早疫、花生褐斑和油菜菌核表现出了很好的防效，化合物 $3 \mathrm{e} \sim 3 \mathrm{o}$ 对花生褐斑、苹果轮纹、番茄早疫、 小麦赤霉、辣椒疫霉、油菜菌核、水稻纹枯等 7 种真菌具有很好的防效, 表现出了广谱的抑菌活性.
\end{abstract}

关键词 1,3-偶极环加成; 异噁唑啉; 合成; 结构表征; 杀菌活性

\section{Synthesis and Fungicidal Activity of Some 3,5-Disubstituted Isoxazoline Derivatives}

\author{
Ning, Guohui ${ }^{a} \quad{\text { Zhao, } \text { Wentao }^{a} \quad \text { Bian, }^{\text {Qiang }}{ }^{b} \quad \text { Tang, Xiangyang }}^{*, a}$ \\ ( ${ }^{a}$ Department of Chemistry, Tianjin University, Tianjin 300072) \\ ( ${ }^{b}$ National Pesticide Engineering Research Center, Nankai University, Tianjin 300071)
}

\begin{abstract}
Various substituted benzaldehydes reacted with hydroxylamine hydrochloride, followed by the chlorination with $\mathrm{N}$-chlorosuccinimide (NCS), to form the corresponding chlorobenzaldehyde oximes, which then underwent the 1,3-dipolar cycloaddition reaction with $N$-vinylpyrrolidone, $N$-vinylpyridine and 4-vinylpyridine, respectively, to generate fifteen novel compounds containing isoxazoline ring. All the title compounds have been characterized by ${ }^{1} \mathrm{H} \mathrm{NMR},{ }^{13} \mathrm{C}$ NMR, and $\mathrm{HRMS}$. Preliminary biological activity tests in vitro indicated that compounds $\mathbf{3 e} \sim \mathbf{3 o}$ displayed excellent fungicidal activity against $C$. arachidicola, $P$. piricola, A. solani, F. graminearum, $P$. capsici, S. sclerotiorum, and $R$. solani in the test concentration, compound $\mathbf{3 b}$ displayed excellent fungicidal activity against $B$. cinerea, and compound $\mathbf{3 c}$ displayed excellent fungicidal activity against $A$. solani, C. arachidicola and $S$. Sclerotiorum.
\end{abstract}

Keywords 1,3-dipolar cycloaddition; isoxazoline; synthesis; structure characterization; fungicidal activity

异噁唑啉类化合物是一类非常重要的杂环化合物, 除其本身可以作为药物使用外, 由于结构中含有 $\mathrm{N}, \mathrm{O}$ 杂环, 为生物碱和相关天然化合物的全合成提供了特别 有效而便捷的途径, 可作为中间体用于合成生物碱 ${ }^{[1]}$. 这类化合物具有杀虫、杀菌、除草、抗病毒和抗惊厥等 多种生物活性, 有些已经被开发成除草剂、杀菌剂、杀 虫剂等农药用品 ${ }^{[2 \sim 6]}$. 比如由美国 Amvac 化学公司与 巴斯夫公司共同开发的除草剂 topramezone(商品名称 Convey), 由美国 FMC 公司开发的除草剂异噁草酮等. 在众多异噁唑衍生物中, 3,5-二取代的异噁唑显示出了 独特的生物活性, 如可以作为潜在的糖蛋白酶 I/II 受体
的拮抗剂, 以及杀菌除草剂, 其中恶霉灵已经实现商品 化 ${ }^{[7 \sim 9]}$. 据报道, 4-乙烯基吡啶聚合物、2-乙烯基吡啶聚 合物、 $N$-乙烯基吡咯烷酮聚合物是聚合物杀菌剂当中很 常见的杀菌剂, 能够有效抑制大部分的细菌或者微生 物 ${ }^{[10,11]}$. 为了寻找具有良好生物活性的异噁唑啉, 我们 将活性功能团吡咯烷酮和吡啶引入异噁唑啉环, 并试着 在异噁唑环的 3-位引入芳基, 经过 1,3-偶极环加成反应 合成了一系列 3,5-二取代的异噁唑啉化合物，以期望得 到生物活性良好的异噁唑啉. 对合成出的化合物的结构 进行了 ${ }^{1} \mathrm{H}$ NMR, ${ }^{13} \mathrm{C}$ NMR 和 HRMS 表征. 初步的生物 活性测试结果表明, 大部分化合物均显示出良好的杀菌

* E-mail: txy@tju.edu.cn

Received March 11, 2014; revised April 19, 2014; published online May 7, 2014.

Project supported by the National Natural Science Foundation of China (No. 21205086).

国家自然科学青年基金(No. 21205086)资助项目. 
活性. 目标化合物的合成路线如 Scheme 1 所示.

\section{1 结果与讨论}

\section{1 目标化合物的合成}

以取代苯甲醛为原料, 采用 Scheme 1 所示的合成 路线, 经 3 步反应制得目的产物. 在此过程中, 取代的 苯甲醛肜及相应的氯代肜的合成, 方法简便且收率较 高. 我们以目标化合物 $\mathbf{3 b}$ 为研究对象, 讨论了缚酸剂对 最后一步 1,3-偶极环加成反应收率的影响. 在相同条件 下, 分别以 $\mathrm{CH}_{3} \mathrm{COOAg}, \mathrm{Et}_{3} \mathrm{~N}, \mathrm{~K}_{2} \mathrm{CO}_{3}$ 为缚酸剂, 选用 $\mathrm{CH}_{2} \mathrm{Cl}_{2}$ 为溶剂, 在 $-5 \sim 0{ }^{\circ} \mathrm{C}$ 条件滴加亲偶极体, 滴加 完之后升至室温, 反应 $18 \mathrm{~h}$, 产物的收率分别为 $70 \%$, $86 \%, 67 \%$. 我们选定 $\mathrm{Et}_{3} \mathrm{~N}$ 为环加成反应的缚酸剂.

\section{2 化合物的图谱分析}

该类化合物的核磁共振图谱比较相似，根据化学位 移, 苯环上的氢处在 $\delta 7.30 \sim 7.67$ 之间, 为多重峰; 五元 杂环 4 位上氢原子的化学位移位于 $\delta 5.84 \sim 6.80$ 之间, 为 $\mathrm{dd}$ 峰; 五元环 5 位上的两个氢原子的化学位移是位于 $\delta 3.07 \sim 3.99$ 之间的多重峰. 而五元环上 3 位上的碳原 子化学位移位于 $\delta 152.7 \sim 161.2$ 之间, 5 位上碳原子化学 位移位于 $\delta 81.5 \sim 83.7$ 之间, 4 位上碳原子的化学位移位 于 $\delta 41.0 \sim 45.0$ 之间.

\section{3 生物活性测试分析}

活性测试结果(表 1)表明: 大部分目标化合物对供 试菌种均表现出了较好的抑菌活性, 对部分菌种抑制活 性达 $100 \%$. 当异噁唑啉环 5 位上的取代基是 1-吡咯烷
酮基时，化合物 $\mathbf{3 b}$ 对黄瓜灰霉表现出了很好的防效，化 合物 $3 \mathrm{c}$ 对番茄早疫、花生褐斑和油菜菌核表现出了很 好的防效; 当 5 位上的取代基是 2-吡啶基或者 4-吡啶基 时，化合物 3e $\sim 30$ 对花生褐斑、苹果轮纹、番茄早疫、 小麦赤霉、辣椒疫霉、油菜菌核、水稻纹枯等七种菌体 均表现出了很好的杀菌效果, 说明其抑菌活性具有良好 的广谱性. 当 5 位上的取代基是吡啶基时, 普遍比 1-吡 咯烷酮基时化合物的抑菌活性高. 目标化合物 $\mathbf{3 d} \sim 3 \mathbf{n}$ 的构效关系表现出一定的规律性, 当有苯环上有 $\mathrm{Cl}$ 取 代基时, 对所有供试真菌均表现出较好的杀菌活性, 普 遍高于其它取代基, 具有进一步的研究价值.

\section{2 结论}

本文通过 1,3-偶极环加成方法合成了 15 个未见报 道的异噁唑啉化合物, 并探究了缚酸剂对反应的影响, 得出环加成反应合成此类 3,5-二取代的异噁唑啉衍生物 合适的反应条件为: 以 $\mathrm{CH}_{2} \mathrm{Cl}_{2}$ 作溶剂, $\mathrm{Et}_{3} \mathrm{~N}$ 为缚酸剂, 在 $-5 \sim 0{ }^{\circ} \mathrm{C}$ 条件下滴加亲偶极体, 室温反应 $18 \mathrm{~h}$; 对 所合成的化合物进行了 ${ }^{1} \mathrm{H} N \mathrm{NMR},{ }^{13} \mathrm{C}$ NMR 鉴定和高分 辨质谱表征. 初步的生物活性测试结果表明, 在试验浓 度下，所有化合物均显示一定的杀菌活性，化合物 $\mathbf{3 b}$ 对 黄瓜灰霉菌表现出了很好地防效, 化合物 $3 \mathrm{c}$ 对番茄早 疫、花生褐斑和油菜菌核表现出了很好的防效, 化合物 3e 30 对花生褐斑、苹果轮纹、番茄早疫、小麦赤霉、 辣椒疫霉、油菜菌核、水稻纹枯这七种真菌表现出了很 好的防效，具有广谱的抑菌活性.
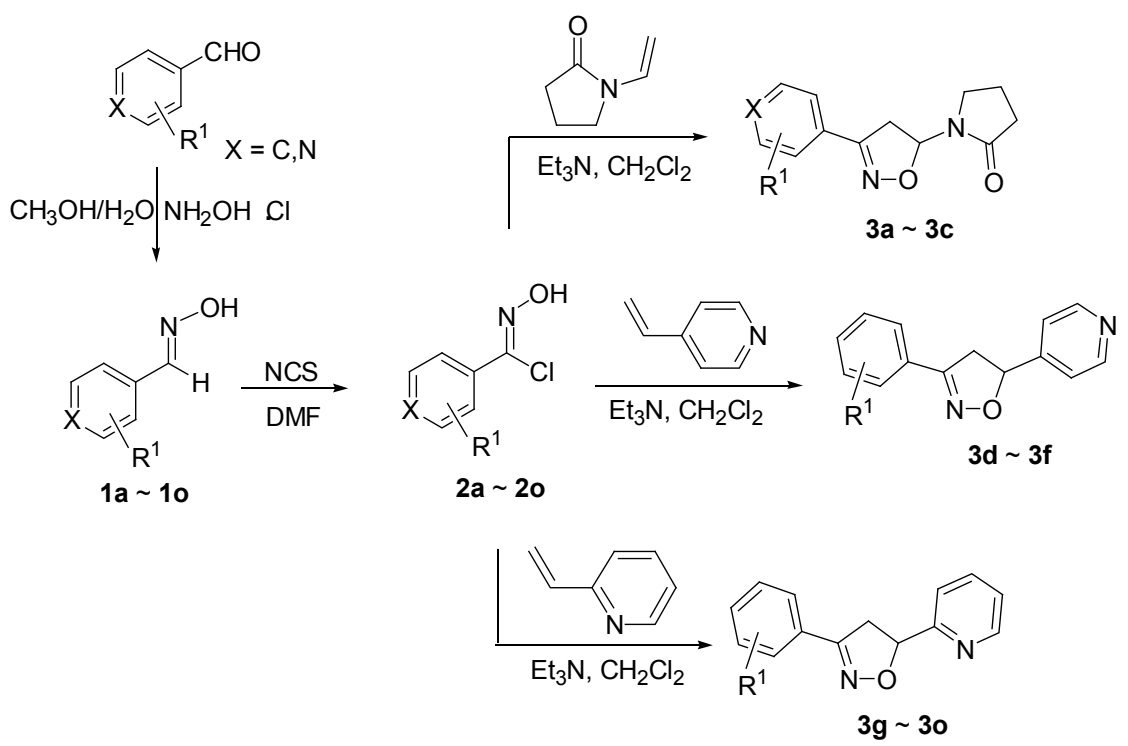

a: $X=C, R^{1}=2-\mathrm{NO}_{2} ; \mathbf{b}: X=2,4-\mathrm{Cl}_{2} ; \mathbf{c}: X=\mathrm{N}, \mathrm{R}^{1}=\mathrm{H} ; \mathbf{d}: \mathrm{R}^{1}=2-\mathrm{NO}_{2} ; \mathbf{e}: \mathrm{R}^{1}=2,4-\mathrm{Cl}_{2} ; \mathrm{f}: \mathrm{R}^{1}=2-\mathrm{CF}_{3} ; \mathbf{g}: \mathrm{R}^{1}=4-\mathrm{NO}_{2} ; \mathbf{h}: \mathrm{R}^{1}=3,4-\mathrm{CH}_{2} \mathrm{O}_{2}$; i: $\mathrm{R}^{1}=2,4-\mathrm{Cl}_{2} ; \mathbf{j}: \mathrm{R}^{1}=2-\mathrm{NO}_{2} ; \mathbf{k}: \mathrm{R}^{1}=4-\mathrm{Cl} ; \mathrm{I}: \mathrm{R}^{1}=3-\mathrm{Cl} ; \mathbf{m}: \mathrm{R}^{1}=2-\mathrm{Cl} ; \mathbf{n}: \mathrm{R}^{1}=2-\mathrm{NO}_{2} ; \mathbf{o}: \mathrm{R}^{1}=4-\mathrm{OCH}_{3}$ 
表 1 目标化合物的离体抑菌活性测试结果 $(50 \mu \mathrm{g} / \mathrm{mL})$

Table 1 The fungicidal activity in vitro of target compounds $(50 \mu \mathrm{g} / \mathrm{mL})$

\begin{tabular}{ccccccccccc}
\hline 化合物 & 黄瓜枯萎 & 花生褐斑 & 苹果轮纹 & 番茄早疫 & 小麦赤霉 & 马铃薯晚疫 & 辣椒疫霉 & 黄瓜灰霉 & 油菜菌核 & 水稻纹枯 \\
\hline $\mathbf{3 a}$ & 26.9 & 29.4 & 58.3 & 23.5 & 47.1 & 32.1 & 36.4 & 34.6 & 26.9 & 36.4 \\
$\mathbf{3 b}$ & 19.2 & 23.5 & 52.8 & 29.4 & 35.3 & 35.7 & 30.3 & 96.2 & 25.0 & 30.3 \\
$\mathbf{3 c}$ & 33.3 & 92.9 & 64.3 & 73.7 & 80.0 & 11.1 & 70.0 & 28.0 & 93.3 & 78.3 \\
$\mathbf{3 d}$ & 19.2 & 23.5 & 61.1 & 29.4 & 64.7 & 39.3 & 45.5 & 26.9 & 17.3 & 37.9 \\
$\mathbf{3 e}$ & 83.3 & 92.9 & 92.9 & 73.7 & 100.0 & 72.2 & 95.0 & 72.0 & 100.0 & 100.0 \\
$\mathbf{3 f}$ & 60.0 & 76.9 & 91.7 & 81.3 & 50.0 & 43.8 & 63.6 & 79.2 & 90.4 & 86.4 \\
$\mathbf{3 g}$ & 22.2 & 100.0 & 85.7 & 68.4 & 80.0 & 33.3 & 95.0 & 36.0 & 86.7 & 73.9 \\
$\mathbf{3 h}$ & 33.3 & 100.0 & 64.3 & 78.9 & 70.0 & 33.3 & 95.0 & 60.0 & 93.3 & 95.7 \\
$\mathbf{3 i}$ & 61.1 & 92.9 & 71.4 & 78.9 & 100.0 & 61.1 & 95.0 & 64.0 & 100.0 & 97.8 \\
$\mathbf{3 j}$ & 16.7 & 92.9 & 64.3 & 63.2 & 90.0 & 16.7 & 80.0 & 32.0 & 76.7 & 87.0 \\
$\mathbf{3 k}$ & 72.2 & 92.9 & 100.0 & 84.2 & 100.0 & 72.2 & 70.0 & 72.0 & 93.3 & 97.8 \\
$\mathbf{3 1}$ & 66.7 & 92.9 & 92.9 & 84.2 & 90.0 & 44.4 & 90.0 & 76.0 & 96.7 & 100.0 \\
$\mathbf{3 m}$ & 66.7 & 85.7 & 78.6 & 78.9 & 100.0 & 55.6 & 95.0 & 64.0 & 100.0 & 93.5 \\
3n & 72.2 & 92.9 & 92.9 & 78.9 & 100.0 & 44.4 & 80.0 & 60.0 & 93.3 & 89.1 \\
$\mathbf{3 0}$ & 22.2 & 100.0 & 14.3 & 73.7 & 90.0 & 22.2 & 90.0 & 48.0 & 93.3 & 87.0 \\
百菌清 & 83.3 & 75.0 & 92.3 & 73.9 & 73.1 & 81.0 & 82.6 & 96.1 & 96.4 & 96.1 \\
\hline
\end{tabular}

\section{3 实验部分}

\section{1 仪器与试剂}

X-4 型数字显示熔点仪 (北京泰克仪器有限公司), 温度计未经矫正; Bruker AVANCE III 400M 型核磁共 振仪 (TMS 为内标, 溶剂为 $\mathrm{CDCl}_{3}$ ); Bruker micrOTOF-Q II 型高分辨质谱仪; 柱层析硅胶为青岛海洋化工厂产品 (200 300 目); 所用溶剂和试剂是分析纯或者化学纯, 必要时需经过干燥或蒸馏处理.

\section{2 合成}

\subsection{1 芳环上取代的芳基苯甲醛肪的合成}

以化合物 $\mathbf{1 b}$ 为例, 在装有机械搅拌、温度计的 100 $\mathrm{mL}$ 反应瓶中加入 $20 \mathrm{~mL}$ 甲醇、 $7.00 \mathrm{~g}(40 \mathrm{mmol}) 2,4-$ 二 氯苯甲醛、 $2.23 \mathrm{~g}(21 \mathrm{mmol})$ 碳酸钠的水溶液, 在搅拌下 向反应体系中分批加入 $3.06 \mathrm{~g}(44 \mathrm{mmol})$ 盐酸羟胺, 控 制反应温度不超过 $30{ }^{\circ} \mathrm{C}, \mathrm{TLC}$ 跟踪反应进程, 反应完全 后, 旋蒸除甲醇, 用乙醚萃取, 有机相用饱和食盐水洗 涤, 再用无水 $\mathrm{MgSO}_{4}$ 干燥, 除去溶剂, 得到化合物 2,4二氯苯甲醛肟 ${ }^{[13]}$, 收率 $89.2 \%$.

\subsection{2中间体氯代芳基苯甲醛肪的合成}

以化合物 $\mathbf{2 b}$ 为例, 在装有磁子、温度计的 $50 \mathrm{~mL}$ 三口瓶中加入 $1.52 \mathrm{~g}(8.0 \mathrm{mmol}) 2,4-$ 二氯苯甲醛肜和 $\mathrm{N}, \mathrm{N}$-二甲基甲酰胺(DMF) $8.0 \mathrm{~mL}$, 在摚拌下分批加入 $1.18 \mathrm{~g}(8.8 \mathrm{mmol}) N$-氯代丁二酰亚胺 $(\mathrm{NCS})$, 控制反应 温度不超过 $45{ }^{\circ} \mathrm{C}, \mathrm{TLC}$ 跟踪反应进程, 反应结束后, 降 至室温, 加水稀释, 再用乙酸乙酯萃取, 有机相用饱和 食盐水洗涤, 再用无水 $\mathrm{MgSO}_{4}$ 干燥, 除去溶剂, 得中间 体粗产品 ${ }^{[13]}$, 粗产品经柱层析提纯, 收率 $83.6 \%$.
3.2.3目标化合物 3,5-二取代异噁唑啉衍生物的合成 以化合物 $\mathbf{3 b}$ 为例, 在装有磁子的反应瓶中加入氯 代 2,4-二氯苯甲醛肪 $1.80 \mathrm{~g}(8.0 \mathrm{mmol}) 、 6 \mathrm{~mL} \mathrm{CH}_{2} \mathrm{Cl}_{2}$ 溶 剂, 在氮气保护下加入 $1.07 \mathrm{~g}(9.6 \mathrm{mmol}) \mathrm{N}$-乙烯基吡咯 烷酮的 $\mathrm{CH}_{2} \mathrm{Cl}_{2}$ 溶液, 保持反应温度在 $-5 \sim 0{ }^{\circ} \mathrm{C}$, 向反 应体系中加入 $1.21 \mathrm{~g}(12.0 \mathrm{mmol}) \mathrm{Et}_{3} \mathrm{~N}$ 的 $\mathrm{CH}_{2} \mathrm{Cl}_{2}$ 溶液, 滴加完毕后室温搅拌, TLC 跟踪反应, 反应结束后用饱 和氯化铵溶液淬灭反应, 然后用 $\mathrm{CH}_{2} \mathrm{Cl}_{2}$ 萃取, 干燥, 除 去溶剂, 产品经柱层析提纯, 得目标化合物 ${ }^{[14,15]}$.

3-(2-硝基苯基)-5-(1-吡咯烷酮基)-2,3-异噁唑啉 (3a): 黄色固体, 收率 75.0\%. m.p. $135.0 \sim 136.8{ }^{\circ} \mathrm{C} ;{ }^{1} \mathrm{H}$ NMR (400 MHz, $\left.\mathrm{CDCl}_{3}\right) \delta: 8.10(\mathrm{~d}, J=8.1 \mathrm{~Hz}, 1 \mathrm{H}), 7.73$ (dd, $J=11.2,3.8 \mathrm{~Hz}, 1 \mathrm{H}), 7.66(\mathrm{t}, J=6.9 \mathrm{~Hz}, 2 \mathrm{H}), 6.73$ (dd, $J=10.3,3.9 \mathrm{~Hz}, 1 \mathrm{H}), 3.51$ (ddd, $J=28.3,16.0,9.1$ $\mathrm{Hz}, 3 \mathrm{H}$ ), $3.23 \sim 3.15$ (m, 1H), 2.47 (t, $J=8.1 \mathrm{~Hz}, 2 \mathrm{H}), 2.12$ $(\mathrm{dd}, J=14.4,6.6 \mathrm{~Hz}, 2 \mathrm{H}) ;{ }^{13} \mathrm{C} \mathrm{NMR}\left(100 \mathrm{MHz}, \mathrm{CDCl}_{3}\right) \delta$ : 175.6, 154.3, 147.9, 133.7, 131.4, 131.0, 124.9,124.9, 83.2, 41.9, 39.0, 31.2, 17.9. HRMS calcd for $\mathrm{C}_{13} \mathrm{H}_{13} \mathrm{~N}_{3} \mathrm{O}_{4} \mathrm{Na}$ $[\mathrm{M}+\mathrm{Na}]^{+}$298.0804, found 298.0798.

3-(2,4-二氯苯基)-5-(1-吡咯烷酮基)-2,3-异噁唑啉 (3b): 白色固体, 收率 85.2\%. m.p. 94.5 96.5 ${ }^{\circ} \mathrm{C} ;{ }^{1} \mathrm{H}$ NMR (400 MHz, $\left.\mathrm{CDCl}_{3}\right) \delta: 7.66(\mathrm{~d}, J=8.4 \mathrm{~Hz}, 1 \mathrm{H}), 7.50$ (d, $J=1.6 \mathrm{~Hz}, 1 \mathrm{H}), 7.34(\mathrm{dd}, J=8.4,1.6 \mathrm{~Hz}, 1 \mathrm{H}), 6.70$ (dd, $J=10.0,3.0 \mathrm{~Hz}, 1 \mathrm{H}), 3.69$ (dd, $J=18.1,10.0 \mathrm{~Hz}, 1 \mathrm{H}$ ), $3.42 \sim 3.27(\mathrm{~m}, 3 \mathrm{H}), 2.45$ (t, $J=8.0 \mathrm{~Hz}, 2 \mathrm{H}), 2.17 \sim 2.01$ $(\mathrm{m}, 2 \mathrm{H}) ;{ }^{13} \mathrm{C}$ NMR $\left(100 \mathrm{MHz}, \mathrm{CDCl}_{3}\right) \delta: 175.4,154.4$, 136.8, 133.4, 131.5, 130.5, 127.7, 126.9, 82.7, 41.9, 39.3, 31.0, 17.8. HRMS calcd for $\mathrm{C}_{13} \mathrm{H}_{12} \mathrm{~N}_{2} \mathrm{O}_{2} \mathrm{Cl}_{2} \mathrm{Na}[\mathrm{M}+\mathrm{Na}]^{+}$ 
321.0174, found 321.0169.

3-(4-吡定基)-5-(1-吡咯烷酮基)-2,3-异噁唑啉(3c): 淡黄色固体, 收率 60.5\%. m.p. 153.2 154.9 ${ }^{\circ} \mathrm{C} ;{ }^{1} \mathrm{H}$ NMR (400 MHz, $\left.\mathrm{CDCl}_{3}\right) \delta: 8.73(\mathrm{~d}, J=5.9 \mathrm{~Hz}, 2 \mathrm{H}), 7.56$ (d, $J=5.9 \mathrm{~Hz}, 2 \mathrm{H}), 6.74(\mathrm{dd}, J=10.1,3.7 \mathrm{~Hz}, 1 \mathrm{H}), 3.55$ (dd, $J=17.7,10.1 \mathrm{~Hz}, 1 \mathrm{H}), 3.35(\mathrm{dd}, J=15.4,8.7 \mathrm{~Hz}, 1 \mathrm{H})$, $3.25 \sim 3.14(\mathrm{~m}, 2 \mathrm{H}), 2.46(\mathrm{t}, J=7.8 \mathrm{~Hz}, 2 \mathrm{H}), 2.13 \sim 2.01$ $(\mathrm{m}, 2 \mathrm{H}) ;{ }^{13} \mathrm{C} \mathrm{NMR}\left(100 \mathrm{MHz}, \mathrm{CDCl}_{3}\right) \delta: 175.4,154.0$, 150.6, 136.1, 120.6, 83.0, 41.7, 36.1, 31.0, 17.8. HRMS calcd for $\mathrm{C}_{12} \mathrm{H}_{13} \mathrm{~N}_{3} \mathrm{O}_{2} \mathrm{Na}[\mathrm{M}+\mathrm{Na}]^{+}$254.0906, found 254.0901 .

3-(2-硝基苯基)-5-(4-吡啶基)-2,3-异噁唑啉(3d): 黄 色固体, 收率 62.3\%. m.p. 124.0 126.0 ${ }^{\circ} \mathrm{C} ;{ }^{1} \mathrm{H}$ NMR $\left(400 \mathrm{MHz}, \mathrm{CDCl}_{3}\right) \delta: 8.68(\mathrm{~d}, J=5.8 \mathrm{~Hz}, 2 \mathrm{H}), 8.10$ (d, $J=$ $8.2 \mathrm{~Hz}, 1 \mathrm{H}), 7.77 \sim 7.69(\mathrm{~m}, 1 \mathrm{H}), 7.64(\mathrm{dd}, J=16.0,8.2$ $\mathrm{Hz}, 2 \mathrm{H}), 7.37(\mathrm{~d}, J=5.7 \mathrm{~Hz}, 2 \mathrm{H}), 5.88 \sim 5.78(\mathrm{~m}, 1 \mathrm{H})$, 3.79 (dd, $J=16.7,11.1 \mathrm{~Hz}, 1 \mathrm{H}), 3.24$ (dd, $J=16.7,7.7 \mathrm{~Hz}$, $1 \mathrm{H}) ;{ }^{13} \mathrm{C} \mathrm{NMR}\left(100 \mathrm{MHz}, \mathrm{CDCl}_{3}\right) \delta: 154.8,150.3,149.1$, 148.0, 133.6, 131.0, 130.9, 124.9, 120.6, 81.5, 45.0. HRMS calcd for $\mathrm{C}_{14} \mathrm{H}_{11} \mathrm{~N}_{3} \mathrm{O}_{3} \mathrm{Na}[\mathrm{M}+\mathrm{H}]^{+}$270.0878, found 270.0872 .

3-(2,4-二氯苯基)-5-(4-吡啶基)-2,3-异啞唑啉(3e): 黄色固体, 收率 63.7\%. m.p. 73.2 75.5 ${ }^{\circ} \mathrm{C} ;{ }^{1} \mathrm{H} \mathrm{NMR}$ $\left(400 \mathrm{MHz}, \mathrm{CDCl}_{3}\right) \delta: 8.66(\mathrm{~d}, J=5.9 \mathrm{~Hz}, 2 \mathrm{H}), 7.65$ (d, $J=$ $8.4 \mathrm{~Hz}, 1 \mathrm{H}), 7.47$ (d, $J=2.0 \mathrm{~Hz}, 1 \mathrm{H}), 7.33$ (dd, $J=13.0$, $4.1 \mathrm{~Hz}, 3 \mathrm{H}), 5.79$ (dd, $J=11.1,7.5 \mathrm{~Hz}, 1 \mathrm{H}), 4.00$ (dd, $J=$ $17.1,11.1 \mathrm{~Hz}, 1 \mathrm{H}), 3.47$ (dd, $J=17.1,7.5 \mathrm{~Hz}, 1 \mathrm{H}) ;{ }^{13} \mathrm{C}$ NMR (100 MHz, $\left.\mathrm{CDCl}_{3}\right) \delta: 155.1,150.3,149.3,136.7$, $133.5,131.4,130.5,127.6,126.9,120.4,81.4,45.0$. HRMS calcd for $\mathrm{C}_{14} \mathrm{H}_{10} \mathrm{~N}_{2} \mathrm{OCl}_{2} \mathrm{Na}[\mathrm{M}+\mathrm{H}]^{+}$293.0248, found 293.0258 .

3-(2-三氟甲基苯基)-5-(4-吡啶基)-2,3-异噁唑啉(3f): 黄色固体, 收率 60.3\%. m.p. 78.6 79.2 ${ }^{\circ} \mathrm{C} ;{ }^{1} \mathrm{H} \mathrm{NMR}$ $\left(600 \mathrm{MHz}, \mathrm{CDCl}_{3}\right) \delta: 8.68 \sim 8.62(\mathrm{~m}, 2 \mathrm{H}), 7.76(\mathrm{~d}, J=7.8$ $\mathrm{Hz}, 1 \mathrm{H}), 7.58$ (ddd, $J=23.7,15.6,7.5 \mathrm{~Hz}, 3 \mathrm{H}), 7.34$ (d, $J=5.8 \mathrm{~Hz}, 2 \mathrm{H}), 5.80(\mathrm{dd}, J=11.1,7.3 \mathrm{~Hz}, 1 \mathrm{H}), 3.86(\mathrm{dd}$, $J=17.2,11.1 \mathrm{~Hz}, 1 \mathrm{H}), 3.27(\mathrm{dd}, J=17.2,7.3 \mathrm{~Hz}, 1 \mathrm{H}) ;{ }^{13} \mathrm{C}$ NMR (101 MHz, $\left.\mathrm{CDCl}_{3}\right) \delta: 155.6,150.3,149.5,132.1$, 130.7, 130.0, 128.1, 126.8, 126.7, 120.4, 81.1, 46.6. HRMS calcd for $\mathrm{C}_{14} \mathrm{H}_{11} \mathrm{~N}_{3} \mathrm{O}_{3} \mathrm{Na}[\mathrm{M}+\mathrm{H}]^{+}$293.0901, found 293.0907.

3-(4-硝基苯基)-5-(2-吡啶基)-2,3-异噁唑啉(3g)：黄 色固体, 收率 82.0\%. m.p. 153.0 155.0 ${ }^{\circ} \mathrm{C}$; ${ }^{1} \mathrm{H}$ NMR $\left(400 \mathrm{MHz}, \mathrm{CDCl}_{3}\right) \delta: 8.62(\mathrm{~d}, J=4.7 \mathrm{~Hz}, 1 \mathrm{H}), 8.28(\mathrm{~d}, J=$ $8.9 \mathrm{~Hz}, 2 \mathrm{H}), 7.89$ (d, $J=8.9 \mathrm{~Hz}, 2 \mathrm{H}), 7.77(\mathrm{td}, J=7.7,1.7$
$\mathrm{Hz}, 1 \mathrm{H}), 7.58$ (d, $J=7.8 \mathrm{~Hz}, 1 \mathrm{H}), 7.30$ (s, 1H), 5.95 (t, $J=$ $9.2 \mathrm{~Hz}, 1 \mathrm{H}), 3.85(\mathrm{~d}, J=9.2 \mathrm{~Hz}, 2 \mathrm{H}) ;{ }^{13} \mathrm{C} \mathrm{NMR}(100 \mathrm{MHz}$, $\left.\mathrm{CDCl}_{3}\right) \delta: 158.8,155.2,149.6,148.5,137.1,135.4,127.6$, 124.0, 123.3, 121.0, 83.5, 40.5. HRMS calcd for $\mathrm{C}_{14} \mathrm{H}_{11^{-}}$ $\mathrm{N}_{3} \mathrm{O}_{3} \mathrm{Na}[\mathrm{M}+\mathrm{H}]^{+}$270.0878, found 270.0872.

3-(3,4-亚甲基二氧苯基)-5-(2-吡啶基)-2,3-异啞唑啉 (3h): 淡黄色晶体, 收率 89.0\%. m.p. 103.2 105.4 ${ }^{\circ} \mathrm{C}$; ${ }^{1} \mathrm{H}$ NMR (400 MHz, $\left.\mathrm{CDCl}_{3}\right) \delta: 8.60(\mathrm{~d}, J=4.7 \mathrm{~Hz}, 1 \mathrm{H})$, $7.73(\mathrm{td}, J=7.7,1.7 \mathrm{~Hz}, 1 \mathrm{H}), 7.59$ (d, $J=7.9 \mathrm{~Hz}, 1 \mathrm{H}), 7.33$ (d, $J=1.5 \mathrm{~Hz}, 1 \mathrm{H}), 7.24(\mathrm{dd}, J=7.0,5.3 \mathrm{~Hz}, 1 \mathrm{H}), 7.08$ (dd, $J=8.1,1.6 \mathrm{~Hz}, 1 \mathrm{H}), 6.83$ (d, $J=8.1 \mathrm{~Hz}, 1 \mathrm{H}), 6.02$ (s, 2H), 5.84 (dd, $J=11.0,6.8 \mathrm{~Hz}, 1 \mathrm{H}), 3.80$ (dd, $J=16.6$, $11.1 \mathrm{~Hz}, 1 \mathrm{H}), 3.67$ (dd, $J=16.6,6.8 \mathrm{~Hz}, 1 \mathrm{H}) ;{ }^{13} \mathrm{C} \mathrm{NMR}$ $\left(100 \mathrm{MHz}, \mathrm{CDCl}_{3}\right) \delta: 160.0,156.1,149.4,149.4,148.1$, $137.0,123.4,122.9,121.8,120.6,108.2,106.6,101.5$, 82.4, 41.7. HRMS calcd for $\mathrm{C}_{15} \mathrm{H}_{12} \mathrm{~N}_{2} \mathrm{O}_{3} \mathrm{Na}[\mathrm{M}+\mathrm{Na}]^{+}$ 291.0746, found 291.0742 .

3-(2,4-二氯苯基)-5-(2-吡啶基)-2,3-异啞唑啉(3i): 淡黄色固体，收率 83.6\%. m.p. 39.3 41.0 ${ }^{\circ} \mathrm{C} ;{ }^{1} \mathrm{H}$ NMR $\left(400 \mathrm{MHz}, \mathrm{CDCl}_{3}\right) \delta: 8.61(\mathrm{~d}, J=4.7 \mathrm{~Hz}, 1 \mathrm{H}), 7.77 \sim 7.76$ (m, $J=7.7,1.7 \mathrm{~Hz}, 1 \mathrm{H}), 7.62(\mathrm{dd}, J=23.8,8.1 \mathrm{~Hz}, 2 \mathrm{H})$, $7.46(\mathrm{~d}, J=2.0 \mathrm{~Hz}, 1 \mathrm{H}), 7.34 \sim 7.22(\mathrm{~m}, 2 \mathrm{H}), 5.89$ (dd, $J=$ 11.0, 7.1 Hz, 1H), 3.99 (dd, $J=17.2,11.0 \mathrm{~Hz}, 1 \mathrm{H}), 3.84$ $(\mathrm{dd}, J=17.2,7.1 \mathrm{~Hz}, 1 \mathrm{H}) ;{ }^{13} \mathrm{C} \mathrm{NMR}\left(100 \mathrm{MHz}, \mathrm{CDCl}_{3}\right) \delta$ : $159.4,155.7,149.5,137.0,136.4,133.6,131.4,130.5$, 127.4, 127.3, 123.1, 120.6, 83.3, 43.5. HRMS calcd for $\mathrm{C}_{14} \mathrm{H}_{10} \mathrm{~N}_{2} \mathrm{OCl}_{2} \mathrm{Na}[\mathrm{M}+\mathrm{Na}]^{+}$315.0068, found 315.0062 .

3-(2-硝基苯基)-5-(2-吡啶基)-2,3-异噁唑啉(3j): 淡 黄色固体, 收率 $86.3 \%$. m.p. $60.8 \sim 62.6{ }^{\circ} \mathrm{C} ;{ }^{1} \mathrm{H}$ NMR $\left(400 \mathrm{MHz}, \mathrm{CDCl}_{3}\right) \delta: 8.62(\mathrm{~d}, J=4.4 \mathrm{~Hz}, 1 \mathrm{H}), 8.06(\mathrm{~d}, J=$ $8.1 \mathrm{~Hz}, 1 \mathrm{H}), 7.87 \sim 7.75(\mathrm{~m}, 1 \mathrm{H}), 7.70(\mathrm{t}, J=7.4 \mathrm{~Hz}, 1 \mathrm{H})$, $7.66 \sim 7.54(\mathrm{~m}, 3 \mathrm{H}), 7.30(\mathrm{~s}, 1 \mathrm{H}), 5.95(\mathrm{dd}, J=11.0,6.9$ $\mathrm{Hz}, 1 \mathrm{H}), 3.78$ (dd, $J=16.8,11.1 \mathrm{~Hz}, 1 \mathrm{H}), 3.61$ (dd, $J=$ $16.8,6.9 \mathrm{~Hz}, 1 \mathrm{H}) ;{ }^{13} \mathrm{C}$ NMR (100 MHz, $\left.\mathrm{CDCl}_{3}\right) \delta: 159.3$, $155.3,149.4,148.1,137.1,133.4,131.1,130.7,125.3$, 124.8, 123.1, 120.8, 83.4, 43.5. HRMS calcd for $\mathrm{C}_{14} \mathrm{H}_{11} \mathrm{~N}_{3} \mathrm{O}_{3} \mathrm{Na}[\mathrm{M}+\mathrm{Na}]^{+} 292.0698$, found 292.0699 .

3-(4-氯苯基)-5-(2-吡啶基)-2,3-异噁唑啉(3k)：黄色 固体, 收率 69.3\%. m.p. 83.0 85.0 ${ }^{\circ} \mathrm{C} ;{ }^{1} \mathrm{H}$ NMR (400 $\left.\mathrm{MHz} \mathrm{CDCl}_{3}\right) \delta: 8.61(\mathrm{~d}, J=4.6 \mathrm{~Hz}, 1 \mathrm{H}), 7.72 \sim 7.76(\mathrm{~m}$, $J=7.7,1.6 \mathrm{~Hz}, 1 \mathrm{H}), 7.65$ (d, $J=8.6 \mathrm{~Hz}, 2 \mathrm{H}), 7.59$ (d, $J=$ $7.8 \mathrm{~Hz}, 1 \mathrm{H}), 7.39$ (d, $J=8.6 \mathrm{~Hz}, 2 \mathrm{H}), 7.26(\mathrm{dd}, J=7.2,5.3$ $\mathrm{Hz}, 1 \mathrm{H}), 5.88$ (dd, $J=11.0,7.0 \mathrm{~Hz}, 1 \mathrm{H}), 3.82$ (dd, $J=16.8$, $11.0 \mathrm{~Hz}, 1 \mathrm{H}), 3.72(\mathrm{dd}, J=16.7,7.0 \mathrm{~Hz}, 1 \mathrm{H}) ;{ }^{13} \mathrm{C} \mathrm{NMR}$ $\left(100 \mathrm{MHz}, \mathrm{CDCl}_{3}\right) \delta: 159.6,155.7,149.4,137.0,136.2$, 
129.0, 128.1, 127.8, 123.1, 120.7, 82.7, 41.2. HRMS calcd for $\mathrm{C}_{14} \mathrm{H}_{11} \mathrm{~N}_{2} \mathrm{OClNa}[\mathrm{M}+\mathrm{Na}]^{+}$281.0458, found 281.0456 .

3-(3-氯苯基)-5-(2-吡啶基)-2,3-异噁唑啉(3I)：呈液 态, 收率 59.0\%. ${ }^{1} \mathrm{H}$ NMR (400 MHz, $\left.\mathrm{CDCl}_{3}\right) \delta: 8.61$ (d, $J=4.5 \mathrm{~Hz}, 1 \mathrm{H}), 7.75(\mathrm{dd}, J=10.9,4.4 \mathrm{~Hz}, 1 \mathrm{H}), 7.70 \sim$ $7.64(\mathrm{~m}, 1 \mathrm{H}), 7.61$ (d, $J=7.8 \mathrm{~Hz}, 1 \mathrm{H}), 7.43$ (d, $J=7.8 \mathrm{~Hz}$, $1 \mathrm{H}), 7.40 \sim 7.29(\mathrm{~m}, 2 \mathrm{H}), 7.25(\mathrm{dd}, J=6.8,5.4 \mathrm{~Hz}, 1 \mathrm{H})$, $5.88(\mathrm{dd}, J=11.0,7.0 \mathrm{~Hz}, 1 \mathrm{H}), 4.01(\mathrm{dd}, J=17.2,11.0 \mathrm{~Hz}$, $1 \mathrm{H}), 3.83$ (dd, $J=17.2,7.0 \mathrm{~Hz}, 1 \mathrm{H}) ;{ }^{13} \mathrm{C} \mathrm{NMR}(100 \mathrm{MHz}$, $\left.\mathrm{CDCl}_{3}\right) \delta: 159.5,155.6,149.4,137.0,134.8,131.1,130.2$, 130.0, 126.9, 124.9, 123.0, 120.7, 82.8, 41.0. HRMS calcd for $\mathrm{C}_{14} \mathrm{H}_{11} \mathrm{~N}_{2} \mathrm{OClNa}[\mathrm{M}+\mathrm{Na}]^{+}$281.0458, found 281.0456.

3-(2-氯苯基)-5-(2-吡啶基)-2,3-异噁唑啉(3m): 呈液 态, 收率 75.3\%. ${ }^{1} \mathrm{H}$ NMR (400 MHz, $\left.\mathrm{CDCl}_{3}\right) \delta: 8.61$ (d, $J=4.5 \mathrm{~Hz}, 1 \mathrm{H}), 7.75$ (t, $J=7.7 \mathrm{~Hz}, 1 \mathrm{H}), 7.70 \sim 7.63(\mathrm{~m}$, $1 \mathrm{H}), 7.61(\mathrm{~d}, J=7.8 \mathrm{~Hz}, 1 \mathrm{H}), 7.43$ (d, $J=7.8 \mathrm{~Hz}, 1 \mathrm{H})$, $7.40 \sim 7.19(\mathrm{~m}, 3 \mathrm{H}), 5.88(\mathrm{dd}, J=11.0,7.0 \mathrm{~Hz}, 1 \mathrm{H})$, $3.90 \sim 3.93(\mathrm{~m}, J=24.2,17.2,9.0 \mathrm{~Hz}, 2 \mathrm{H}) ;{ }^{13} \mathrm{C}$ NMR $(100$ $\left.\mathrm{MHz}, \mathrm{CDCl}_{3}\right) \delta: 159.5,155.6,149.5,137.0,134.8,131.1$, $130.1,130.0,126.9,124.9,123.0,120.7,82.8,41.0$. HRMS calcd for $\mathrm{C}_{14} \mathrm{H}_{11} \mathrm{~N}_{2} \mathrm{OCINa}[\mathrm{M}+\mathrm{Na}]^{+} 281.0458$, found 281.0458 .

3-(3-硝基苯基)-5-(2-吡啶基)-2,3-异噁唑啉(3n): 白 色固体, 收率 73.3\%. m.p. 96.0 98.0 ${ }^{\circ} \mathrm{C} ;{ }^{1} \mathrm{H}$ NMR (400 $\left.\mathrm{MHz}, \mathrm{CDCl}_{3}\right) \delta: 8.63(\mathrm{~d}, J=4.7 \mathrm{~Hz}, 1 \mathrm{H}), 8.51(\mathrm{~s}, 1 \mathrm{H})$, $8.33 \sim 8.23(\mathrm{~m}, 1 \mathrm{H}), 8.12(\mathrm{~d}, J=7.8 \mathrm{~Hz}, 1 \mathrm{H}), 7.76(\mathrm{td}, J=$ 7.7, $1.6 \mathrm{~Hz}, 1 \mathrm{H}), 7.69 \sim 7.57(\mathrm{~m}, 2 \mathrm{H}), 7.30(\mathrm{~s}, 1 \mathrm{H}), 5.96(\mathrm{~d}$, $J=9.1 \mathrm{~Hz}, 1 \mathrm{H}), 3.87(\mathrm{~d}, J=9.1 \mathrm{~Hz}, 2 \mathrm{H}) ;{ }^{13} \mathrm{C}$ NMR $(100$ $\left.\mathrm{MHz}, \mathrm{CDCl}_{3}\right) \delta: 158.9,155.0,149.6,148.5,137.1,132.4$, $131.2,129.8,124.6,123.3,121.7,120.9,83.2,40.6$. HRMS calcd for $\mathrm{C}_{14} \mathrm{H}_{11} \mathrm{~N}_{3} \mathrm{O}_{3} \mathrm{Na}[\mathrm{M}+\mathrm{Na}]^{+}$292.0698, found 292.0697.

3-(4-甲氧基苯基)-5-(2-吡啶基)-2,3-异噁唑啉(3o): 黄色固体, 收率 85.2\%. m.p. 69.3 71.0 ${ }^{\circ} \mathrm{C} ;{ }^{1} \mathrm{H}$ NMR $\left(400 \mathrm{MHz}, \mathrm{CDCl}_{3}\right) \delta: 8.60(\mathrm{~d}, J=4.7 \mathrm{~Hz}, 1 \mathrm{H}), 7.73$ (td, $J=7.7,1.7 \mathrm{~Hz}, 1 \mathrm{H}), 7.66(\mathrm{~d}, J=8.8 \mathrm{~Hz}, 2 \mathrm{H}), 7.61$ (d, $J=$ $7.7 \mathrm{~Hz}, 1 \mathrm{H}), 7.24$ (dd, $J=6.8,5.3 \mathrm{~Hz}, 1 \mathrm{H}), 6.93$ (d, $J=8.8$ $\mathrm{Hz}, 2 \mathrm{H}), 5.84(\mathrm{dd}, J=11.0,6.7 \mathrm{~Hz}, 1 \mathrm{H}), 3.88 \sim 3.80(\mathrm{~m}$, $4 \mathrm{H}), 3.69(\mathrm{dd}, J=16.7,6.7 \mathrm{~Hz}, 1 \mathrm{H}) ;{ }^{13} \mathrm{C} \mathrm{NMR}(100 \mathrm{MHz}$, $\left.\mathrm{CDCl}_{3}\right) \delta: 161.2,160.2,156.1,149.3,137.0,128.4,122.9$, $121.8,120.6,114.1,82.2,55.4,41.8$. HRMS calcd for $\mathrm{C}_{15} \mathrm{H}_{14} \mathrm{~N}_{2} \mathrm{O}_{2} \mathrm{Na}[\mathrm{M}+\mathrm{Na}]^{+}$277.0953, found 277.0948.

\section{3 生物活性测试}

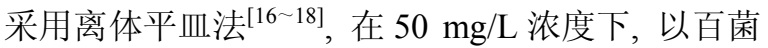
清为对照药, 对黄瓜枯萎病菌(Fusarium oxysporium),
花生褐斑病菌(Cercospora arachidicola), 苹果轮纹病菌 (Physalospora piricola), 番茄早疫病菌 (Alternaria solani), 小麦赤霉病菌(Fusarium graminearum), 马铃薯 晚疫病菌 (Rhizoctoniasolani), 辣椒疫霉病菌 (Phytophthora capsici), 黄瓜灰霉病菌(Botrytis cinerea), 油菜菌核病菌(Sclerotinia Sclerotiorum), 水稻纹枯病菌 (Rhizoctonia solani)十种菌体做杀菌活性试验. 以加入 等体积无菌水的培养基为空白对照, 十字交叉法量取菌 落直径，通过下式计算抑制率.

抑制率 $(\%)=($ 对照菌落扩散直径一处理菌落扩散 直径) $/$ (对照菌落扩散直径 $) \times 100 \%$

辅助材料(Supporting Information) 所合成化合物的 ${ }^{1} \mathrm{H}$ NMR, ${ }^{13} \mathrm{C} \mathrm{NMR}, \mathrm{HRMS}$ 谱图. 这些材料可以免费从 本刊网站(http://sioc-journal.cn/)上下载.

\section{References}

[1] Cheng, C. S.; Li, Z. N.; Su, J. Y.; Li, T.; Zhang, B. Y. Chin. J. Org. Chem. 2005, 25, 1392 (in Chinese).

(程春生，李志念，苏金燕，李涛，张宝砚，有机化学，2005，25, 1392.)

[2] Dubios, R. J.; Lin, C. C.; Beisler, J. A. J. Med. Chem. 1978, 21, 301.

[3] Clive, B. C. B.; Heme, B.; Shirley, B. W. US 4066770, 1978 [Chem. Abstr. 1978, 87, 23258a].

[4] Ahaber, S. H.; Zhang, L. X.; Szapacs, E. M.; Quinn, J. A. EP 1035122, 2002 [Chem. Abstr. 2002, 133, 222723].

[5] Barbudan, M. R.; Morris, J.; Wishka, D. O. US 6069141, 2000 [Chem. Abstr. 2000, 131, 170354].

[6] Al-Abed, Y. US 7662843, 2010 [Chem. Abstr. 2010, 138, 33331]

[7] Fan, Y. J.; He, T. T.; Yang, J. C.; Liu, C. L. Agrochem. Res. Appl. 2010, 14, 1 (in Chinese).

(范玉杰, 赫形形, 杨吉春, 刘长令, 农药研究与应用, 2010, 14 , 1.)

[8] He, H. W.; Li, M. Q.; Huang, G. L. Pesticide 2000, 39, 4 (in Chinese). (贺红武, 李美强, 黄刚良, 农药, 2000, 39, 4.)

[9] Wityak, J.; Sielecki, T. M.; Pinto, D. J.; Emmett, J.; Sze, J. Y.; Liu, J.; Tobin, A. E.; Wang, S. G.; Jiang, B.; Ma, P.; Mousa, S. A.; Olson, R. E.; Wexler, R. R. J. Med. Chem. 1997, 40, 50.

[10] Gao, B. J.; He, S. X.; Guo, J. F.; Wang, R. X. Mater. Lett. 2007, 61, 877.

[11] Stratton, T. R.; Applegate, B. M.; Youngblood, J. P. Biomacromolecules 2011, 12, 50.

[12] Blecher, L.; Lorenz, D. H.; Lowd, H. L.; Wood, A. S.; Wyman, D. P. In Handbook of Water-Soluble Gums and Resins, Ed.: Davidson, R. L., McGraw-Hill Book Company, New York, 1980, pp. $21 \sim 22$.

[13] Liu, K. C.; Shelton, B. R.; Howe, R. K. J. Org. Chem. 1980, 45, 3917.

[14] Boruah, M.; Konwar, D. Synth. Commun. 2012, 42, 3261.

[15] Houk, K. N.; Sims, J.; Watts, C. R.; Luskus, L. J. Am. Chem. Soc. 1973, 95, 7301.

[16] Funaki, Y.; Ishiguri, Y.; Kato, T.; Tanaka, S. J. Pestic. Sci. 1984, 9 , 229.

[17] Chen, N. C. Bioassay of Pesticides, Beijing Agricultural University Press, Beijing, 1991, pp. 161 162 (in Chinese). 
(陈年春, 农药生物测定技术, 北京农业大学出版社, 北京, 1991, pp. 161 162.)

[18] Dai, H.; Miao, W. K.; Liu, J. B.; Wu, Sh. Sh.; Qin, X.; Fang, J. X.
Chin. J. Org. Chem. 2012, 32, 1690 (in Chinese).

(戴红, 苗文科, 刘建兵, 吴珊珊, 秦雪, 方建新, 有机化学, 2012, 32, 1690.)

(Qin, X.) 